PoS $\quad \begin{aligned} & \text { PROCEEDINGS } \\ & \text { OF SCIENCE }\end{aligned}$

\title{
Measurements of Higgs boson production and properties in the di-lepton decay channel using the CMS detector
}

\section{Pooja Saxena*t}

Deutsches Elektronen-Synchrotron, Notkestrasse 85, 22607 Hamburg, Germany

E-mail: pooja.saxena@desy.de

The most recent results from CMS on the search for a neutral Higgs bosons decaying into a tau or a muon pair will be presented.

38th International Conference on High Energy Physics

3-10 August 2016

Chicago, USA

*Speaker.

${ }^{\dagger}$ On behalf of CMS collaboration 


\section{Introduction}

On 4 July 2012, the ATLAS and CMS experiments at CERN's Large Hadron Collider announced they had each observed a new particle in the mass region around $125 \mathrm{GeV}[1,2,3]$. Since then, many precise measurements of these properties have been performed: the latest mass measurement, the signal strength, and the couplings with bosons ( $\gamma \gamma, \mathrm{ZZ}$ and WW) [4, 5]. All these measurements are consistent with the Higgs boson predicted by the Standard Model (SM) [4, 5].

However, to claim that this newly observed particle is the SM Higgs boson, the couplings to leptons has to be verified as well. At the LHC, the Higgs boson can be produced in several ways, mainly by gluon-gluon fusion (ggH), also by Vector Boson Fusion (VBF), in association with a $\mathrm{W} / \mathrm{Z}$ boson $(\mathrm{VH})$, or in association with a top quark pair (ttH). It mostly decays into a pair of bquarks (57\%), in a pair of two $\tau$ leptons $(6.3 \%)$, and more rare into a pair of muons $(0.02 \%)$. In this document, results from Run-I on measurements involving Higgs boson decays to leptons $(\tau \tau$, $\mu \mu$ ) will be reviewed in section 2. Measurement of Higgs boson with lepton coupling is discussed in section 3. Further, measurements on universality of Higgs boson coupling flavour is address in section 4. A search for high mass Higgs boson of the MSSM decaying into leptons using the first 2015 data at $13 \mathrm{TeV}$ is presented in section 5. Finally, conclusions are given in section 6

\section{Higgs ( $125 \mathrm{GeV})$ decay to leptons}

Among the Higgs boson decay to a di-lepton pair, the $\tau \tau$ is the one with the largest branching ratio (BR), because of the large event rate expected in the SM compared to the other leptonic decay modes. This section reports on the results of a search for a SM Higgs boson using final states with a pair of $\tau$ leptons in proton-proton collisions at $\sqrt{s}=7$ and $\sqrt{s}=8 \mathrm{TeV}$ at the LHC. Entire dataset collected in 2011 and 2012 by the CMS experiment corresponding to an integrated luminosity of $4.9 \mathrm{fb}^{-1}$ at a centre of mass energy of $7 \mathrm{TeV}$ and $19.7 \mathrm{fb}^{-1}$ at $8 \mathrm{TeV}$ is being used [7]. All the possible decay modes of the di-tau system are used: $e \mu, e e, \mu \mu, e \tau_{h}\left(\tau_{h}\right.$ represent the hadronic decay mode of $\tau$ ), $\mu \tau_{h}$ and $\tau_{h} \tau_{h}$. The main backgrounds are represented by $Z \rightarrow \tau \tau, Z \rightarrow l l$, $\mathrm{W}+\mathrm{jets}$ events and QCD processes. For most of the channels, the signal extraction is done with a likelihood fit on the di-tau mass. Events are categorized exploiting the number of jets, boost of the Higgs system and the sensitivity to ggH production according to the expected S/B. The "0jet" categories are mostly used to constrain the main backgrounds. The combined observed and predicted $m_{\tau \tau}$ distributions for the $\mu \tau_{h}, e \tau_{h}, \tau_{h} \tau_{h}$, and $e \mu$ channels is shown in Fig 1 (left). An excess of events over the background-only hypothesis is observed with a local significance of 3.2 standard deviations at $m_{H}=125 \mathrm{GeV}$, while the expected significance is 3.7 standard deviations and the best-fit value for the signal strength, $\mu=\sigma / \sigma_{S M}$, is $0.78 \pm 0.27$ times the SM prediction, see Fig 1 (right), from which it can be conclude that there is evidence of the Higgs boson decays to pair of $\tau$ lepton.

\section{Measurement of Higgs lepton coupling}

Fig 2 shows fits for deviations in the coupling for a generic five-parameter model assuming effective loop couplings according to the SM. For the fermions, the values of the fitted Yukawa 

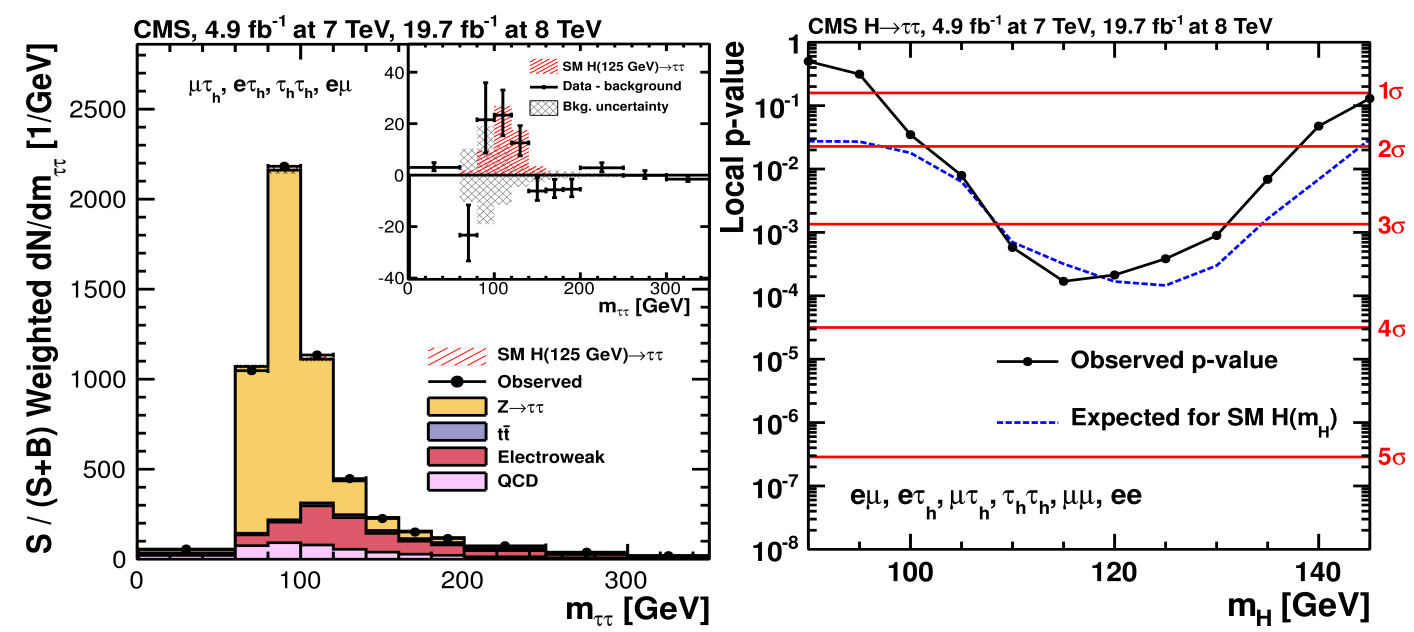

Figure 1: Combined observed and predicted $m_{\tau \tau}$ distributions for the $\mu \tau_{h}, e \tau_{h}, \tau_{h} \tau_{h}$, and $e \mu$ channels (left). Local p-value and significance in number of standard deviations as a function of the SM Higgs boson mass hypothesis (right)

couplings are shown, while for vector bosons the square-root of the coupling for the hVV vertex divided by twice the vacuum expectation value of the Higgs boson field is shown. The linear dependence on the mass parameter nicely illustrates the validity of the Higgs mechanism providing the masses of the fundamental particles in the SM.

\section{Is Higgs lepton coupling flavour Universal?}

In this section, measurement of Higgs boson coupling to first and second generation leptons will be presented [12]. The analysis is performed by directly combining the full $\sqrt{s}=7 \mathrm{TeV}$ and $\sqrt{s}=8 \mathrm{TeV}$ datasets $\left(5.0+19.7 \mathrm{fb}^{-1}\right)$, using the ggH and VBF production modes (the crosssection times $\mathrm{BR}$ is too small for the $\mathrm{VH}$ and $\mathrm{ttH}$ production mode). The $\mathrm{SM}$ predicts the coupling of the new boson to leptons to be proportional to the lepton mass: for $H \rightarrow \mu \mu$ is very low $(2.2 \times$ $10^{-4}$ ), and even smaller for $H \rightarrow e e$. Nonetheless, the signature of these decays is very clean, although affected by a large DY background.

Events are categorized based on the number or reconstructed jets attempting to separate the gluon-gluon fusion and VBF production components. The signal is extracted by means of a fit to the $m_{\mu \mu}$ distribution using signal and background shapes. As no significant $H \rightarrow \mu \mu$ is observed, upper limits on the cross section times the $H \rightarrow \mu \mu$ branching ratio are set. The combined observed (expected) limit on the signal strength, for a Higgs boson with a mass of $125 \mathrm{GeV}$, is $7.4\left(6.5_{-1.9}^{+2.8}\right)$, Fig. 3 (left). Assuming the SM production cross section, this corresponds to an upper limit of 0.0016 on $\beta(H \rightarrow \mu \mu)$. For $H \rightarrow e e$, SM Higgs boson decays are far too rare to detect, and no signal is observed. For a Higgs boson mass of $125 \mathrm{GeV}$, a $95 \% \mathrm{CL}$ upper limit of $0.041 \mathrm{pb}$ is set on $\sigma \beta(H \rightarrow e e)$ at $8 \mathrm{TeV}$, see Fig. 3 (right).

These results, together with the above quoted evidence for the $125 \mathrm{GeV}$ boson coupling to $\tau$ leptons confirm the SM prediction that the leptonic couplings of the new boson are not flavour universal. 


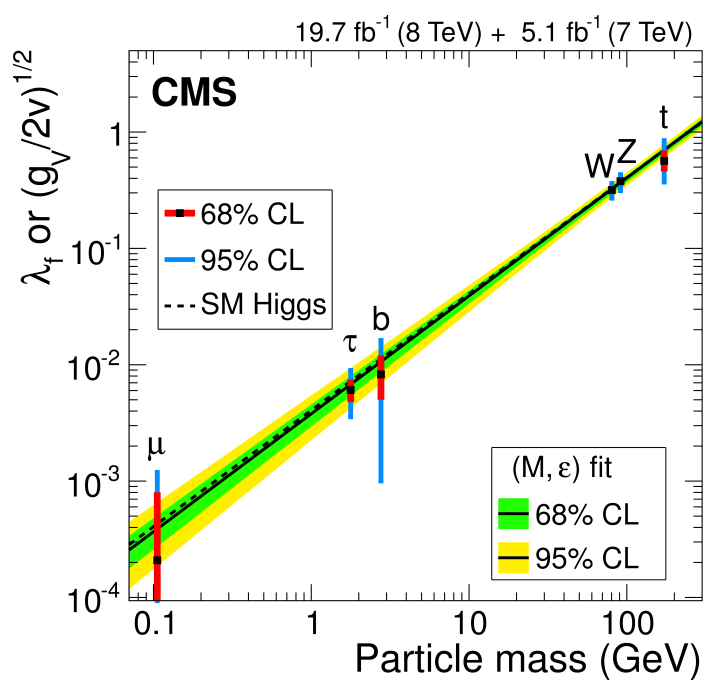

Figure 2: Summary of the fits for deviations in the coupling using the SM loop induced couplings, expressed as function of the particle mass.
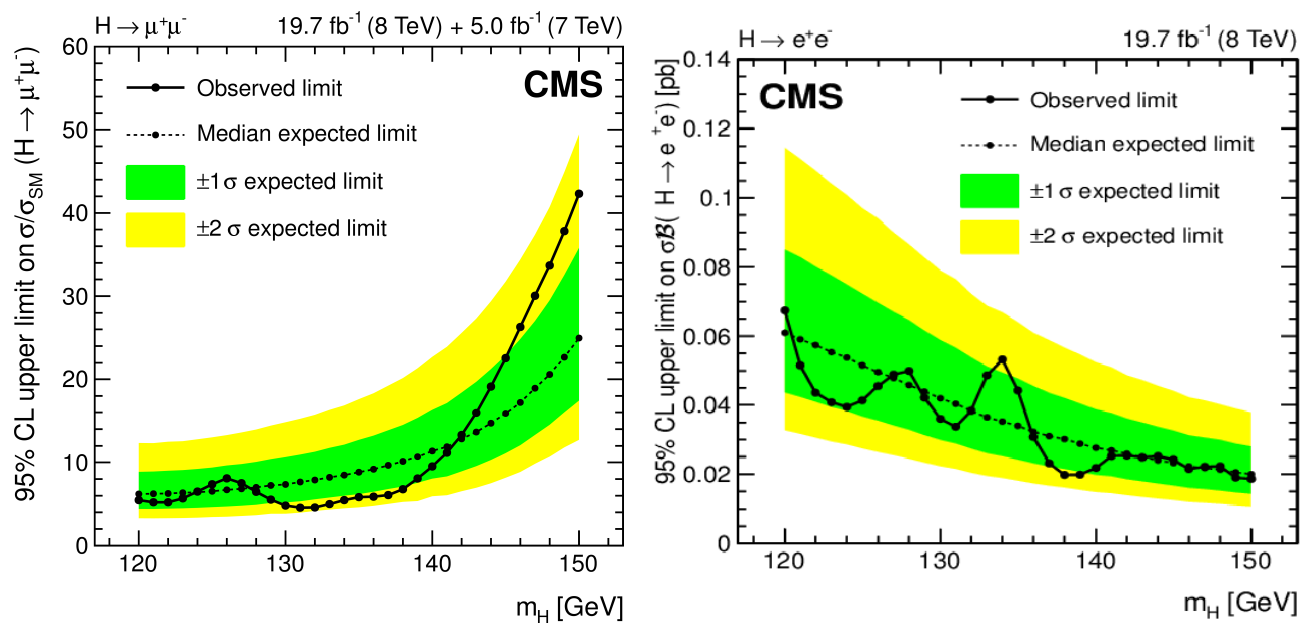

Figure 3: Exclusion limits on $\sigma \beta$ are shown for $H \rightarrow \mu \mu$ (left), and for $H \rightarrow e e$ (right).

\section{Higgs results at $13 \mathrm{TeV}$}

MSSM is the simplest extension of SM, include an extended Higgs sector and favored by many theoretical arguments [13]. This search is performed on a dataset corresponding to an integrated luminosity of $2.3 \mathrm{fb}^{-1}$ of pp collision data at a centre-of-mass energy of $13 \mathrm{TeV}$, collected by CMS in 2015.

The trigger situation was challenging in Run-II compared to Run-I. Fig. 4 show the efficiency of L1 and HLT as a function of offline $\tau p_{T}$ for data and simulation [14]. The analysis strategy is close to the Run-I, though the physics objects, selection and analysis tools are re-optimized. For example, improved multivariate analysis (MVA) based discriminant using the information of combine isolation and lifetime [15]. Fig. 5 shows the performance improvement of MVA over cut-based for probability of mis-identification as a function of $\tau_{h}$ identification efficiency. Also, 

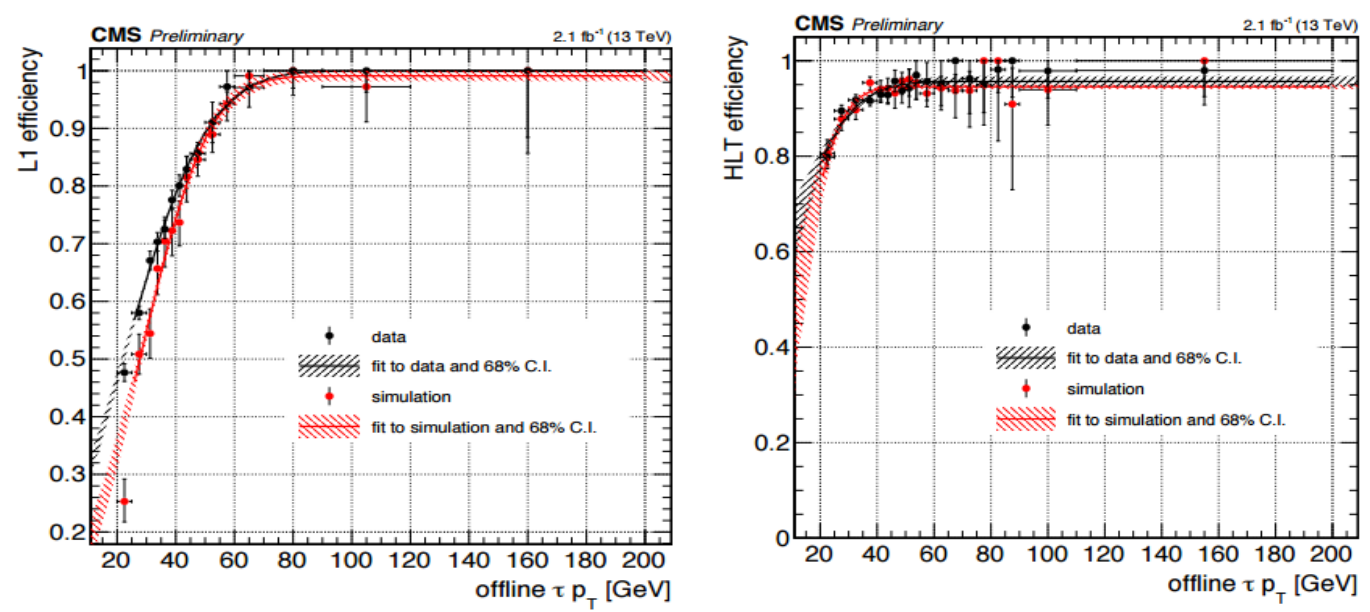

Figure 4: L1 efficiency as a function of offline $\tau p_{T}$ (left), HLT efficiency as a function of offline $\tau p_{T}$ (right).

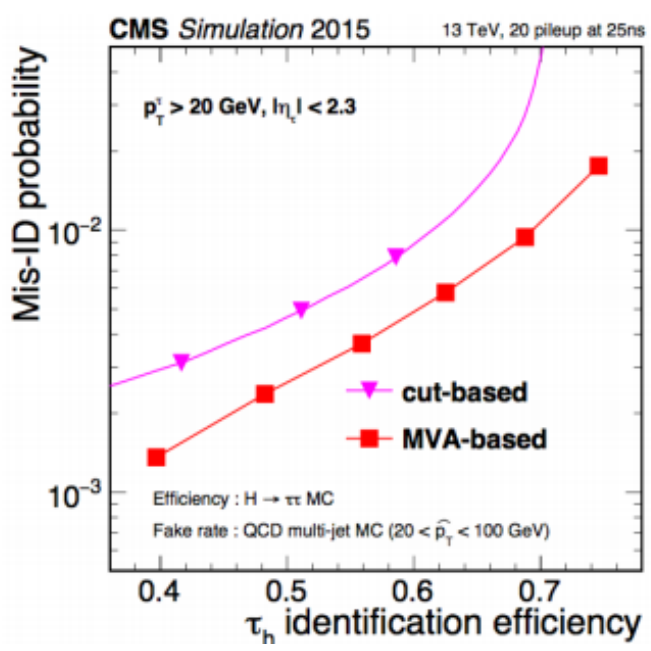

Figure 5: Probability of mis-identification vs $\tau_{h}$ identification efficiency for cut based and for MVA based selection.

improved tau reconstruction and identification using the information of new strip reconstruction algorithm was introduced, where ECAL energy leakage of $\tau_{h}$ decay was accounted [15].

Four final states of the tau pair are considered: $e \mu, e \tau_{h}, \mu \tau_{h}$ and $\tau \tau$. The dominant production mode is gluon fusion (ggf) and b-associated production (bbf). A binned maximum likelihood fit is performed simultaneously to the transverse mass distributions in the b-tag and no b-tag categories and all four channels Fig. 6. As no significant excess of data over the background predictions is found, we set $95 \%$ CL upper limits on cross-section times branching fraction for the two dominant production modes, ggf and bbf. Fig. 7 shows these limits as a function of $m_{\phi}$. These results are shown for different values of the Higgs boson mass $m_{\phi}$. Finally, Fig. 8 shows the model dependent limits for two different benchmark models, the $m_{h}^{\bmod +}$ and hMSSM scenarios. 

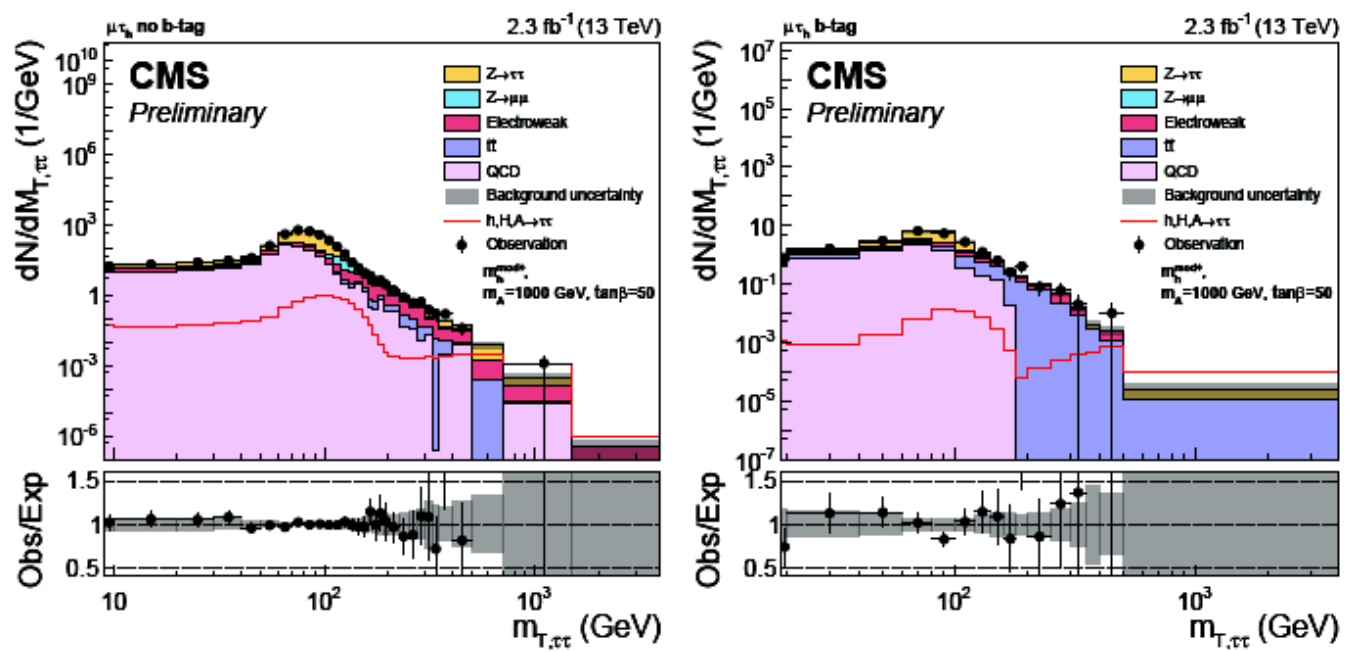

Figure 6: Post-fit plot of the transverse mass distribution in the no b-tag category (left) and the b-tag category of the $e \tau_{h}$ channel (right).
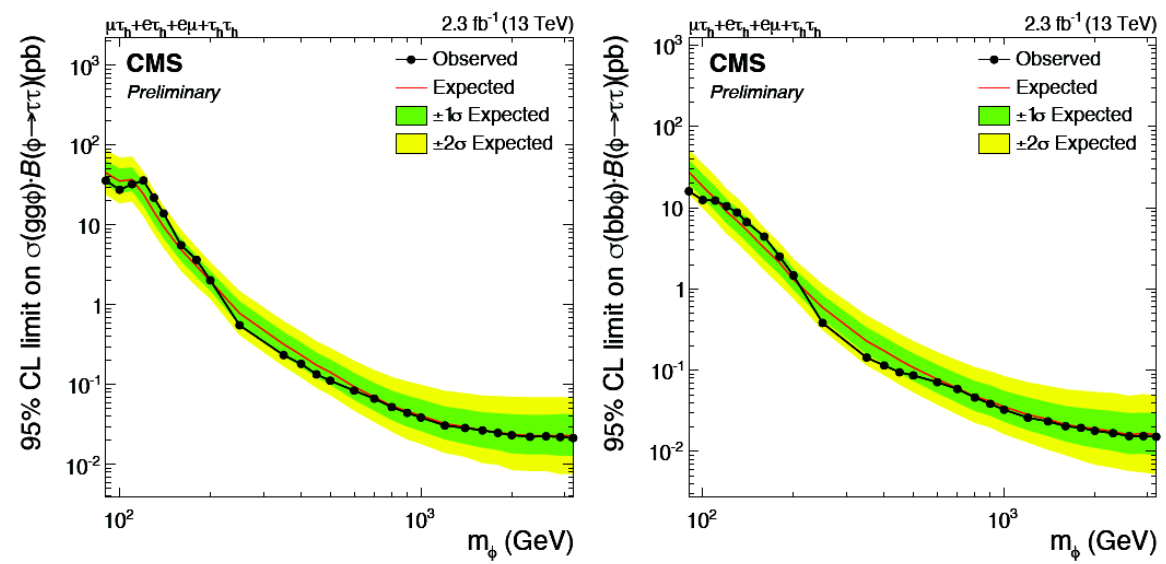

Figure 7: Expected and observed limits on cross-section times branching fraction for the gluon fusion process (left) and the b-associated production process (right), resulting from the combination of all four channels.

\section{Summary}

Measurements of SM Higgs boson production and properties in the di-lepton decay channel have been performed in CMS for different channels $(\tau \tau, \mu \mu)$, using different production modes. Results of Minimal Supersymmetric Standard Model (MSSM) analysis of Higss to ditau as a stepping stone towards Higgs to ditau measurement in Run-II are shown. No significant excess of data over the background predictions is found, limits in various scenarios are drawn. 

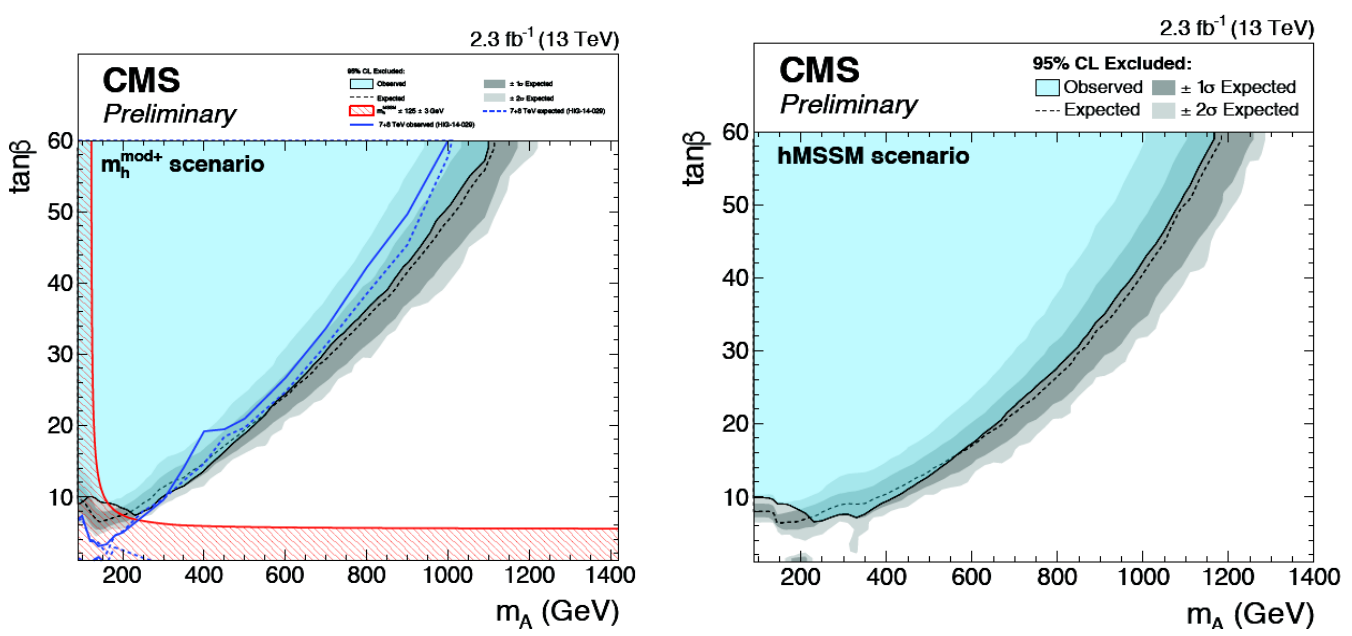

Figure 8: Model dependent exclusion limits in the $m_{A}-\tan \beta$ plane, combining all channels, for the $m_{h}^{\text {mod }+}$ (left) and hMSSM (right) scenarios.

\section{References}

[1] CMS Collaboration, Phys. Lett. B716 30 (2012).

[2] CMS Collaboration, JHEP06 081 (2013) .

[3] ATLAS Collaboration, Phys. Lett. B716 1 (2012).

[4] CMS Collaboration, JHEP01 096 (2014).

[5] CMS Collaboration, Phys. Rev. D89 092007 (2014).

[6] CMS Collaboration, JINST3 S08004 (2008)

[7] CMS collaboration, J. High Energ. Phys. 104 (2014), doi:10.1007/JHEP05(2014)104

[8] LHC Higgs Cross Section Working Group, CERN Report CERN-2012-002, 2012. 534 doi:10.5170/CERN-2012-002, arXiv:1201.3084.

[9] CMS Collaboration, CMS-PAS-HIG-16-003.

[10] CMS collaboration, Nature Physics 10, 557-560 (2014) doi:10.1038/nphys3005

[11] CMS Collaboration, CMS-PAS-HIG-16-005.

[12] CMS Collaboration, Phys. Lett. B744 (2015) 184-207

[13] CMS Collaboration, CMS-PAS-HIG-16-006.

[14] CMS collaboration, detector performance note, DP-2016/037

[15] CMS collaboration, detector performance note, DP-2016/019 